\section{Distribution of NADPH-diaphorase and AChE activity in the anterior leaflet of rat mitral valve}

\author{
K. Lovasova, D. Kluchova, A. Bolekova, \\ F. Dorko, T. Spakovska \\ Department of Anatomy, Faculty of \\ Medicine, P.J. Safarik University, Kosice, \\ Slovak Republic
}

\section{Abstract}

The mitral valve, as an active flap, forms the major part of the left ventricular inflow tract and therefore plays an important function in many aspects of left ventricular performance. The anterior leaflet of this valve is the largest and most ventrally placed of two leaflets that come together during ventricular systole to close the left atrioventricular orifice. Various neurotransmitters are responsible for different functions including controlling valve movement, inhibiting or causing the failure of impulse conduction in the valve and the sensation of pain. Nitric oxide acts as a gaseous free radical neurotransmitter, neuromediator and effective cardiovascular modulator. Acetyl-choline is known to function as a typical neurotransmitter. Histochemical methods for detection of nicotinamide adenine dinucleotide phosphate diaphorase (NADPH-d), as an indirect nitric oxide-synthase marker, and method for detection of acetylcholinesterase (AChE) were used. Both methods were performed on the same valve sample. A widespread distribution of nerve fibres was observed in the anterior leaflet of the mitral valve. The fine NADPH-d positive (nitrergic) nerve fibres were identified in all zones of valve leaflet. AChE positive (cholinergic) nerve fibres were identified forming dense network and fibres organized in stripes. Endocardial cells and vessels manifested heavy NADPH-d activity. Our observations suggest a different arrangement of nitrergic and cholinergic nerve fibres in the anterior leaflet of the mitral valve. The presence of nitrergic and cholinergic activity confirms the involvement of both neurotransmitters in nerve plexuses and other structures of mitral valve.

\section{Introduction}

Heart valves are critical structures in the function of the heart, and the surgical repair of diseased valves has become a major form of treatment for patients with valve disease.
Paradoxically, little is known about the structural components that are critical factors in the function of the heart valves. ${ }^{1}$ However, the evidence linking valve innervation with functional integrity of the heart valves or establi-shing correlations between innervation and valve dysfunction is meagre. ${ }^{2}$ From the anatomical and physiological points of view, the anterior leaflet of the mitral valve (anterior mitral leaflet, or the anterior cusp of the left atrioventricular valve) forms an important boundary of the left outflow tract. ${ }^{3}$ This might imply that this structure is a possible candidate for a special sensory area among the leaflets ${ }^{2,4}$ and it is shown to be a highly active structure in the beating heart. ${ }^{5}$ The two leaflets of the mitral valve are actively contractile; the physical forces generated in the valve itself may stabilize and add precision to the sum of forces regulating valve movement. This is a critical significance both in the moments preceding and during the valve opening and closing. ${ }^{5,6}$

Although the function alteration of the cardiac autonomic nervous system has been proved to be a powerful predictor of cardiac death or serious arrhythmia in patients with cardiac disease, little is known yet about the mechanisms regulating this system. Despite the increasing evidence about the importance of cardiac autonomic function abnormalities in heart disease, the controlling mechanisms remain poorly understood. ${ }^{?}$

The mammalian and human hearts contain a variety of morfologically distinct nerve fibres known to influence cardiac function. Just the cardiac valve tissue has been shown to be innervated by nerve fibres containing active sympathetic, parasympathetic and peptidergic neurotransmitters. ${ }^{4,8}$ Recent studies ${ }^{7,9-12}$ suggest that nitric oxide (NO) may play an important role in the control of this regulatory system and that neural regulation of coronary blood flow and cardiac conductance is essential for the maintenance of cardiac function. ${ }^{13}$

NO as a gaseous free radical acts like a neurotransmitter and effective cardiovascular modulator. This gas plays a fundamental role in cardiovascular physiology and pathophysiology. ${ }^{14,15}$ Within the cardiovascular system, NO participates in the regulation of coronary blood flow and tension of vessel wall. ${ }^{11,16}$ It is released not only from endothelial cells (ECs), but also may serve as a non-adrenergic, non-cholinergic neurotransmitter in cardiac innervation. ${ }^{7,11}$ The regulators of NO production are physical and chemical stimulators transmitted by the vessel wall and by endocardium as well. NO influences all neural substrates that contribute to the generation of the autonomic nerves activity in the central and peripheral nervous system. ${ }^{9,17-20} \mathrm{NO}$ is synthesized from its precursor L-arginine by the enzyme NO synthase (NOS), which occurs in three distinct iso-
Correspondence: Kvetuse Lovasova, Department of Anatomy, Faculty of Medicine, P.J. Safarik University, Srobarova 2, 04001 Kosice, Slovak Republic. Tel.: +421.903662976.

E-mail: kvetuse.lovasova@upjs.sk

Key words: NADPH-diaphorase, acetylcholinesterase, heart, mitral valve, nerve fibres, vessels, rat.

Acknowledgements: this work was supported by VEGA grant 1/0643/08

Received for publication: 5 November 2009.

Accepted for publication: 7 January 2010.

This work is licensed under a Creative Commons Attribution 3.0 License (by-nc 3.0).

CCopyright K. Lovasova et al., 2010

Licensee PAGEPress, Italy

European Journal of Histochemistry 2010; 54:e5 doi:10.4081/ejh.2010.e5

forms. These consist of the constitutive calcium-dependent enzymes endothelial NOS (eNOS, originally identified in vascular endothelium, but also present in platelets, myocardium and endocardium) and neuronal NOS (nNOS, present in neuronal tissue) and the calcium-independent, cytokine-inducible NOS (iNOS, expressed in macrophages and other tissues during immune stimulation).$^{10}$ In the vascular and cardiac tissue it is thus constitutively produced by eNOS or nNOS, which has been shown to be identical with NADPHdiaphorase under condition of tissue fixation. Reduced NADPH-d may be used as a marker for NOS, since NOS and NADPH-d are extensively co-localized..$^{21}$ Although the NADPH-d reaction is not completely specific, it is nonetheless highly selective for NOS activity in the heart. There are no other diaphorases to confound localization in this organ as an evidence of the reduction of nitro blue tetrazolium (NBT) to the formazan insoluble in water. ${ }^{22}$

NO is described also as a neurotransmitter and neuromodulator in the vagus nerve, similarly to an acetylcholine. ${ }^{11}$ It has additional effects on the level of parasympathetic postganglionic nerve terminals that may be particularly important within the heart. Many studies suggest that NO modulates cardiac vagal control, increase the activity of central vagal motoneurons and, more contentiously, contribute to the bradycardic effects of vagal stimulation. Overall, a picture of NO as a sympatholytic and a vagotonic agent does emerge from the animal data. ${ }^{7}$

Acetylcholine (ACh) is known to function as a typical and primary chemical neurotransmitter. Although both divisions of the autonomic nervous system are crucial for the heart regulation, the anatomic distribution of cholinergic 
(parasympathetic) nerves is less precisely known than that of sympathetic innervation. $\mathrm{AChE}$ is an important component of the heart's cholinergic system; it is known to regulate the cardiac parasympathetic responses by controlling acetylcholine levels. ${ }^{23}$ According to Williams and Jew, ${ }^{6}$ the rat mitral valve shows that the contractions and relaxations of valves are nerve-mediated.

Therefore, the purpose of the present study was to confirm and compare the distribution of both NADPH-d and AChE enzymes in the anterior mitral leaflet of the rat heart. The presence and especially the mutual relationship of both enzymes can contribute to understanding of $\mathrm{NO}$ and ACh neurotransmitters in functioning of mitral valves.

\section{Materials and Methods}

\section{Groups of animals used in experiment}

Twenty male white Wistar rats, aged 3 months, with a mean weight of 250-350 g were used in this study. Rats were housed in cages and maintained under standard conditions at $20^{\circ} \mathrm{C}$, with $12 \mathrm{~h}$ light/dark cycle and water and food ad libitum. All experiments were conducted in accordance with European Directive for the Protection of Vertebrate Animals Used for Experimental and Other Scientific Purposes $(86 / 609 / \mathrm{EU})$ and approved by the State Veterinary and Food Administration of Slovak Republic by decision 1696/07-221a.

\section{Samples of tissue and fixation}

Animals were anaesthetized with pentobarbital $(50 \mathrm{mg} / \mathrm{kg})$. A midline abdominal incision, followed by two lateral costal margin incisions exposed the diaphragm, which was then incised. The thoracic cavity was opened and the heart was perfused with a saline solution and $4 \%$ paraformaldehyde with $0.1 \%$ glutaraldehyde in $0.1 \mathrm{M}$ phosphate buffer (PB), $\mathrm{pH}$ 7.4. The heart was removed, the left ventricle was rapidly opened and the valves were cut from the atrioventricular ring. The sample material was placed in the same fixative for 2 hours. The fixed tissue was transferred overnight into $30 \%$ saccharose in the same $\mathrm{PB}$ at $4^{\circ} \mathrm{C}$. For double staining of NADPH-d and $\mathrm{AChE}$, the valves were first stained for NADPHd histochemistry and as follows for AChE histochemistry. Both methods were performed separately on the same valve preparations.

\section{NADPH-diaphorase and AChE histochemistry}

The investigation of NADPH-diaphorase activity structures of the anterior leaflet of the

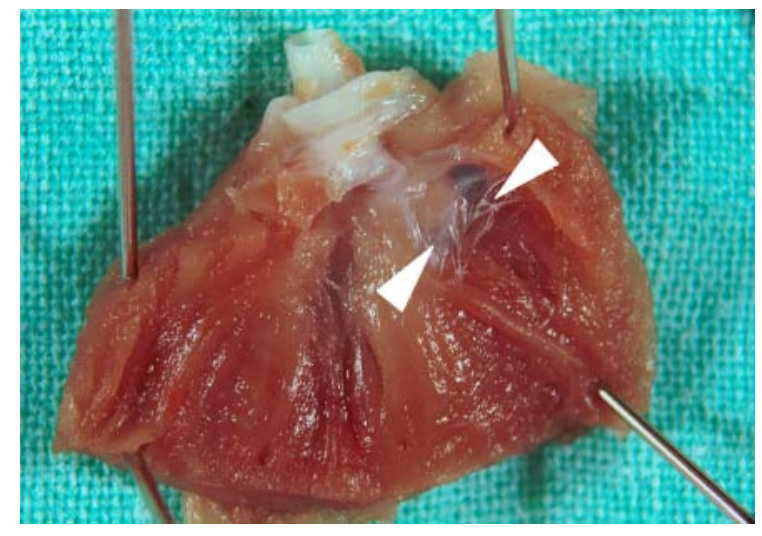

Figure 1. The mitral valve of the rat heart. The picture shows the zone adjacent to the fibromuscular ring (arrow on the left) and the free moving zone (arrow on the right), (original magnification $\times 5$ ).

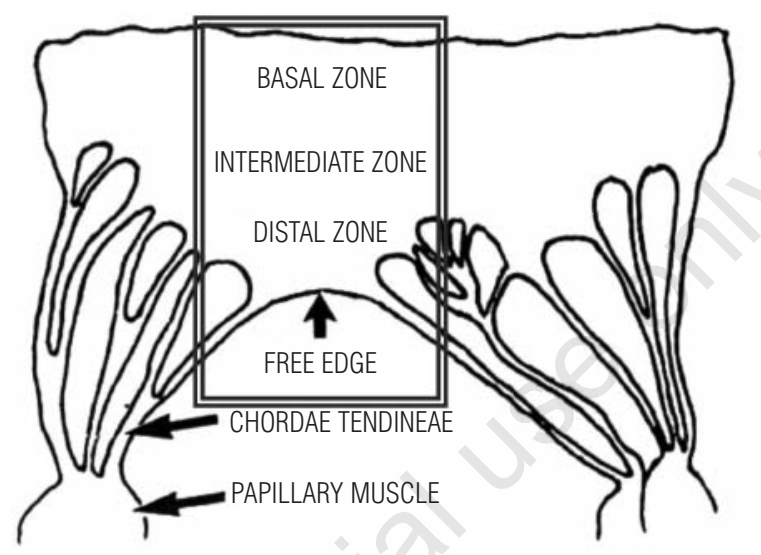

Figure 2. Scheme of the mitral valve. Drawing of the valve segment depicting the basal, intermediate and distal zones (arranged according to Ahmed et al. $)^{33}$ left mitral valve was carried out using an histochemical technique according to SchererSingler et $a l .^{24}$ and modified in our laboratory conditions..$^{25}$ The valves were incubated for $1 \mathrm{~h}$ at $37^{\circ} \mathrm{C}$ in a solution containing $1.5 \mathrm{mM} / \mathrm{L}$ nitroblue tetrazolium (NBT, Sigma Chemicals, $\mathrm{N}-6876), \quad 1.0 \quad \mathrm{mM} / \mathrm{L} \quad \beta-\mathrm{NADPH} \quad($ Sigma Chemicals, N-1630), $10.0 \mathrm{mM} / \mathrm{L}$ monosodium malate (Malic acid, Sigma Chemicals, M-1125) and $0.5 \%$ Triton X-100. The reaction product was seen as a dark blue stained structures. The study by Dawson et al. ${ }^{21}$ has confirmed that under condition of tissue fixation the NADPHdiaphorase is identical with the NO synthase. Our laboratory conditions were in accordance with that study (the valves were fixed by paraformaldehyde).

The AChE positive nerve structures were visualised by a direct histochemical thiocholine method according to El-Badawi and Schenk. ${ }^{26}$ The tissue was incubated in a staining solution. Iso-OMPA was used to selectively block the enzymatic activity of AChE. The doubly stained valves were rinsed in distilled water, mounted on glass slides by a wholemount stretch technique, dried overnight and covered with Entellan (Merck, Germeny). The preparations were observed under a light microscope Optica B600 TI with photo Moticam 2300 and Motic images Plus, 2.0 ML.

\section{Results}

For descriptive purposes, the innervation of the mitral valve (anterior mitral leaflet) was divided into 3 zones. The basal zone lies close to the fibromuscular (atrioventricular) ring attachment of the valves. The intermediate zone is in the middle third of the mitral valve. The distal zone lies close to the free edge of the valve and attachment of the chordae tendineae; it is a free moving zone of the mitral valve (Figures 1,2).

In whole-mount preparations of the mitral valves, the majority of NADPH-d staining was seen in the anterior leaflet only. Under physiological condition with various intensity, vessels (Figures $3 \mathrm{~A}, \mathrm{C}$ ), fine nerve fibres (Figures $3 \mathrm{C} ; 4 \mathrm{C} ; 5 \mathrm{~A}, \mathrm{~B})$ and endothelial cells of the endocardium (Figures 3A,C; 4A,B; 5A,B) in the examined leaflet were stained from light blue to dark blue colour.

NADPH-d positive arterioles and capillares were localized in the attachment (basal) zone of the anterior mitral leaflet (Figures $3 \mathrm{~A}, \mathrm{C}$ ). The fine NADPH-d positive (nitrergic) nerve fibres were identified in basal zone running close to the vessels and in the tunica adventitia as well (Figure 3C). Most endocardial (endothelial) cells (EECs) were only lightly NADPH-d stained in basal zone, but a small 


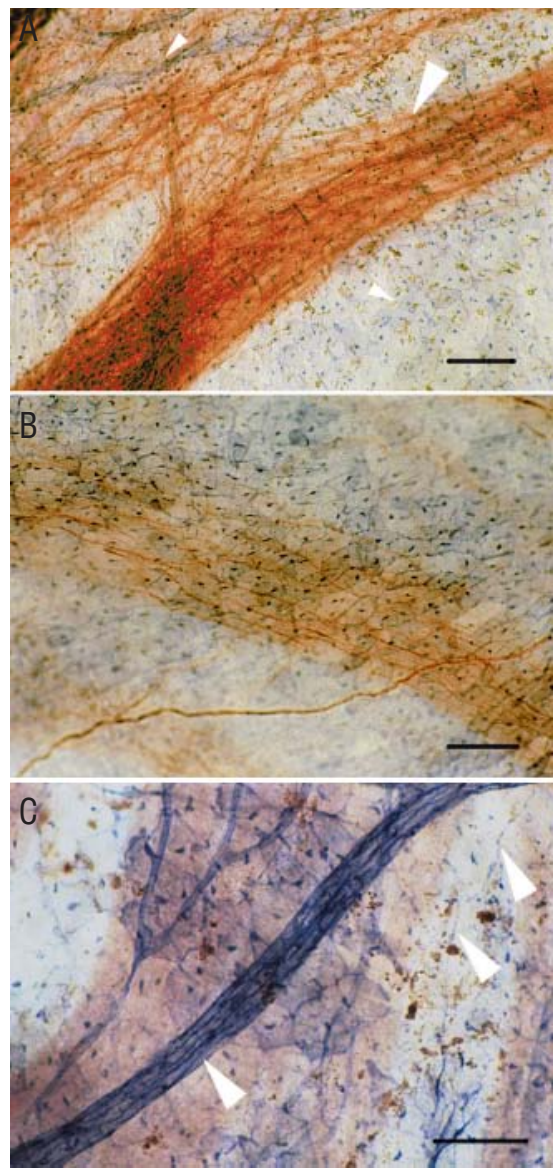

Figure 3. (A, B, C). The basal zone of the anterior mitral leaflet. NADPH-d positive arterioles and capillares are localized in the area of fibromuscular ring (3A: small arrow up; 3C: arrow on the left). The fine NADPH-d positive (nitrergic) nerve fibres running close to the vessels (3C: arrows on the right) and in tunica adventitia (arrow on the left). Blue stained endothelial cells are seen in different colour on the background (3A: small arrow down; 3C). AChE positive (cholinergic) nerve fibres are identified forming both a dense network and fibres organized in stripes (3A: big arrow; 3B). Calibration bars: 3A, B $20 \mu \mathrm{m}, 3 \mathrm{C} 10 \mu \mathrm{m}$.

amount of EECs and nerve fibres were stained moderately in this zone (Figures 3A,B,C).

In the intermediate zone and especially in the distal zone, nitrergic nerve fibres were running along margins of EECs or crossing them only in the blood flow direction (Figures $4 \mathrm{C} ; 5 \mathrm{~A}, \mathrm{~B})$. The fibres reached neither the free margin of the valve leaflet nor the chordae tendineae. Heavy NADPH-d activity was manifested by the EECs edges and probably the perinuclear cytoplasm as well. The cytoplasm showed the staining intensity from medium to very light (Figures 4A, B; 5A, B). Especially, in the central part of the distal zone of the valve leaflets, EECs were elongated and had a polygonal shape (Figure 5A,B). The cells in the
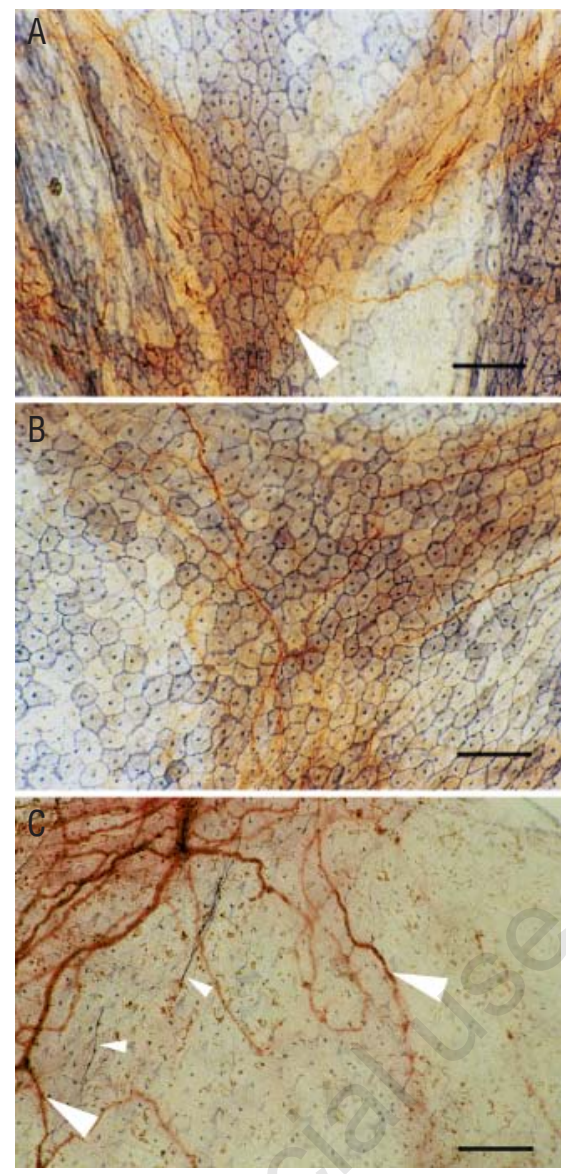

Figure 4. (A, B, C). The intermediate zone of the anterior mitral leaflet. The fine NADPHd positive nerve fibres (4C: small arrows) and blue stained endothelial cells are seen in different colour on the background. $\mathrm{AChE}$ positive nerve fibres are in this area sparsely arranged (4A: arrow; 4B; 4C: big arrows). Calibration bars: 4A, B, C $20 \mu \mathrm{m}$.

basal and intermediate zones (Figures 3A,C; $4 A, B)$ were less elongated compared with the ones in the distal zone.

By means of the histochemical method, the NADPH-d activity of EECs in the anterior mitral leaflet, endothelium of vessels presented in it and nerve fibres was confirmed by indirect determination of the eNOS and nNOS synthases activity.

AChE positive structures were seen stained amber yellow. In spite of the direct course of NADPH-d positive nerve fibres, AChE positive nerve fibres were found running in various directions. Network of cholinergic nerve enters from the area of fibromuscular (atrioventricular) ring and extends predominantly into the basal and intermediate zones of the valve leaflet. AChE positive nerve fibres were identified first of all in basal zone forming both a dense network and fibres organized in stripes (Figures 3A,B). Intermediate and distal
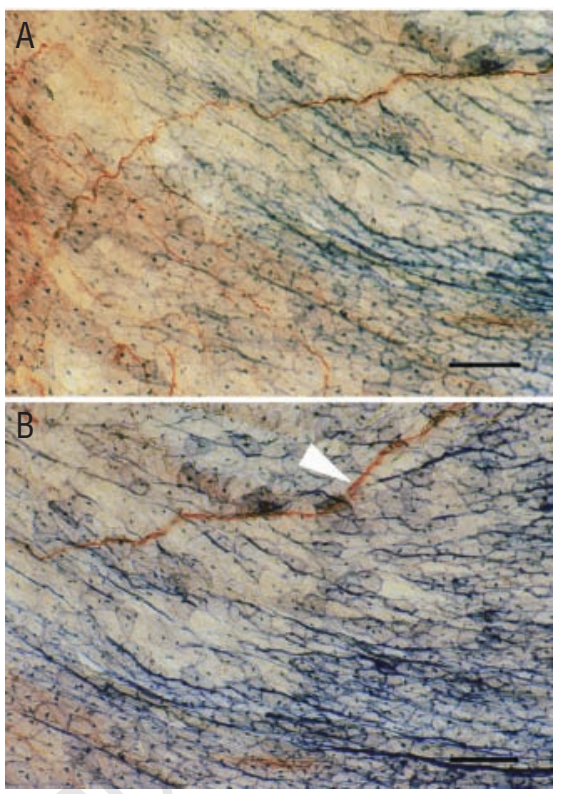

Figure 5. (A, B). The distal zone of the anterior mitral leaflet. The fine NADPH-d positive nerve fibres were identified especially in this zone running along margins of endothelial (endocardial) cells or crossing them only in the direction of blood flow. In spite of the direct course of NADPH-d positive nerve fibres, $\mathrm{AChE}$ positive nerve fibres were seen running in various directions (5B: arrow; 5A). Calibration bars: 5A, B $20 \mu \mathrm{m}$.

zones of anterior mitral leaflet revealed AChE positive nerve fibres sparsely arranged (Figures 4A,B,C; $5 \mathrm{~A}, \mathrm{~B})$. They took up $2 / 3-3 / 4$ of the valve leaflet size. No cholinergic nerve fibres reached lower parts of the distal zone or the chordae tendineae.

\section{Discussion}

It is well known that the mammalian heart displays regional distribution of autonomic innervation, whereby branches of sympathetic and parasympathetic nerves influence on certain cardiac regions in a different extent, ${ }^{6,27}$ also depending on individual age and health status. ${ }^{28-30}$ The occurrence and distribution of different neurotransmitters and neuromediators were investigated in the heart in different species of animals by investigating the presence of acetylcholine, ${ }^{23,31}$ nitric oxide, neuropeptide $\mathrm{Y}$, tyrosine hydroxylase and calcitonin gene related peptide. ${ }^{1,31-34}$

The co-localization of NADPH-d and nNOS in mitral valve leaflets was characterized also by Olsen et al. ${ }^{15}$ Reports by Ursell and Mayes, ${ }^{22}$ Shoba and Tay, ${ }^{35}$ Kukanova and Mravec $^{27}$ have studied the localization, histology and function of cardiac neurones that contain a marker for NADPH-d and NOS. There is also strong histo- 
chemical evidence for the presence of NOS throughout cardiac autonomic neurones. Cardiac innervation is further complicated by the presence of intrinsic neurones, which are known to be capable of significant interaction with both efferent and afferent (sensory) neurones. ${ }^{31}$ The NADPH-d activity in subpopulation of cardiac neurones was reported by Maifrino et al. ${ }^{36} \mathrm{NADPH}-\mathrm{d}$ positive neurones in the heart could provide another source of NO, in addition to endothelial and endocardial cells, and contribute to the actions of NO on the heart. This is an evidence, that NO supports and plays a role in the local control of the heart by means of intrinsic neurones. ${ }^{37}$

The most relevant physiological stimuli for the release of $\mathrm{NO}$ from endocardial endothelial cells may be flow-induced shear stress and the cyclical mechanical deformation of the endocardium that occurs during the cardiac cycle.,14 The most obvious structural response of endothelium to shear stress is to change cell shape and its orientation. ${ }^{9,38,39}$ Endocardium of rat is characterized by a very thin subendocardial layer of connective tissue. As stated above, this may cause possible alteration in shape and orientation of EECs. The blood flow can function as a stress factor to the valve surface, especially in the free moving part of the valve leaflet.

The presence of strongly positive NADPH-d reaction was determined in the blood vessels of rat heart by Ursell and Mayes. ${ }^{22}$ NADPH-d positive fibres were relatively abundant also in the sinus node, but were very sparse in the atrioventricular node and in the right bundle branch. ${ }^{40}$ Nevertheless, it is possible that NADPH-d reactive nerve fibres have direct influence on the function of the valves, the frequency and force of valve closure and the cardiac contraction; they may play a role in precipitating arrhythmias and premature extrasystoles. ${ }^{15,41}$ An accurate and full understanding of the precise movements of the valve leaflets and the mechanisms regulating these movements is likely to provide the information to understanding and development of treatment for many different cardiac valve problems, including mitral valve diseases such as prolapse and myxomatous degeneration. ${ }^{15}$

It is generally known, that previous studies. ${ }^{26,31,42,43}$ have used acetylcholinesterase (AChE) histochemistry to identify cholinergic nerves in the heart. Cholinergic nerve terminals were localized predominantly in endocardial plexuses of the atria and in the left ventricle. According to Williams et al., ${ }^{42}$ the nerve plexus of the atrioventricular valve is possessing primary, secondary and tertiary components and this terminology was applied in more recent studies. ${ }^{1,33}$

At this time, we have no complete information of both nitrergic and cholinergic innerva- tion, nitrergic and cholinergic co-expression in rat heart valves. Howewer, the main functional role of nitric oxide in peripheral cholinergic modulation is known. ${ }^{44,45}$ The study of Yoshida and Toda ${ }^{46}$ in the intracardiac plexus of monkey and canine presents also the colocalization of NOS-positivity and AChE in neurones. Some small neurones were intensely stained for NADPH-d activity, but they were not co-localized with AChE. Our observations confirmed both different localization of NADPH-d and AChE positive nerve fibres and their different course in regions of rat valve leaflet. These findings indicate different targets and functions of neurotransmitters in these nerve structures. The demarcation between regions of the valve innervated by cholinergic versus nitrergic nerve fibres may also correlate with the line of leaflet closure. The anterior leaflet of mitral valve, as a neurally-controlled tissue, provides thus a clinically relevant model for the investigation of functional role of nervous system in normal and abnormal heart valve action. ${ }^{1,5,30}$

Our observations confirmed different arrangement of nitrergic and cholinergic nerve fibres in the anterior leaflet of mitral valve in rat. The presence of nitrergic and cholinergic activity in nerve plexuses of atrioventricular valve confirms their involvement in modulation of neurotransmission. These findings can contribute to further study of valve innervation in some cardiac diseases (valves stenosis and insufficiency). Our results suggest that nitric oxide and acetylcholine may also play a role as neurotransmitters and/or neuromediators in neural control of the cardiac blood flow and impulse conduction.

\section{References}

1. Jew JY, Fink CA, Williams TH. Tyrosine hydroxylase-and nitric oxide synthaseimmunoreactive nerve fibers in mitral valve of young adult and aged Fischer 344 rats. J Autonom Nerv Syst 1996;58:35-43.

2. Fann JI, Ingels NB, Miller DC. Pathophysiology of mitral valve disease. In: Cohn LH, Edmunds LH Jr., (eds.), Cardiac Surgery in the Adult. McGraw-Hill, New York, 2003; pp. 901-931.

3. Kachlik D, Baca V, Bozdechova I et al. Anatomical terminology and nomenclature: past, presence and highlights. Surg Rad Anat 2008;30:459-66.

4. Yacoub MH, Cohn LH. Novel approaches to cardiac valve repair: From structure to function: Part I. Circulation 2004;109:94250.

5. Itoh A, Krishnamurthy G, Swanson JC et al. Active stiffening of mitral valve leaflets in the beating heart. Am J Physiol 2009;296:1766-73.

6. Williams TH, Jew JY. Is the mitral valve passive flap theory overstated? An active valve is hypothesized. Med Hypotheses 2004;62:605-11.

7. Chowdhary S, Townend JN. Role of nitric oxide in the regulation of cardiovascular autonomic control. Clin Sci 1999;97:5-17.

8. Massion PB, Feron 0, Dessy C, Balligand JL. Nitric oxide and cardiac function. Ten years after, and continuing. Circ Res 2003;93:388-98.

9. Sears CE, Ashley EA, Casadei B. Nitric oxide control of cardiac function: is neuronal nitric oxide synthase a key component? Philos Trans R Soc Lond B Biol Sci 2004;359:1021-44.

10. Naseem KM. The role of nitric oxide in cardiovascular disease. Mol Aspects Med 2005;26:33-65.

11. Roy P, Venkat RG, Naidu MUR, Usha RP. Recent trends in the nitrergic nervous system. Educational Forum 2005;37:69-76.

12. Jones SP, Bolli R. The ubiquitous role of nitric oxide in cardioprotection. $\mathrm{J}$ Mol Cell Cardiol 2006;40:16-23.

13. Llorens S, Jordan J, Nava E. The nitric oxide pathway in the cardiovascular system. J Physiol Biochem 2002;58:179-88.

14. Shah AM, Vallance P, Harrison D. NO in the cardiovascular system. Cardiovasc Res 1999; 43:507-8.

15. Olsen LH, Mortensen T, Martinussen T et al. Increased NADPH-diaphorase activity in canine myxomatous mitral valve leaflets. J Comp Pathol 2003;129:120-30.

16. Paulus WJ, Shah AJ. NO and cardiac diastolic function. Cardiovasc Res 1999;43: 595-606.

17. Zanzinger J. Role of nitric oxide in the neural control of cardiovascular function. Cardiovasc Res 1999;43:639-49.

18. Kluchova D, Rybarova S, Miklosova M et al. Comparative analysis of NADPHdiaphorase positive neurons in the rat, rabbit and pheasant thoracic spinal cord. A histochemical study. Eur J Histochem 2001;45:239-48.

19. Kluchova D, Kloc P, Klimcik R et al. The effect of long-term reduction of aortic blood flow on spinal cord gray matter in the rabbit. Histochemical study of NADPHdiaphorase. Cell Mol Neurobiol 2006;26: 1253-64.

20. Sulla I, Vanicky I, Balik V. Sustained versus short-term cauda equina constrictions result in a selective damage of lumbosacral spinal cord neuronal pools in the dog. Folia Veterinaria 2008;52:113-8.

21. Dawson TM, Bredt TS, Fotuhi M et al. Nitric oxide synthase and neuronal NADPH-diaphorase are identical in brain 
and peripheral tissues. Proc Natl Acad Sci USA, 1991;88:7779-801.

22. Ursell P, Mayes M. Anatomic distribution of nitric oxide synthase in the heart. Cardiovasc Res 1995;50:217-23.

23. Hoover DB, Ganote ChE, Ferguson SM et al. Localization of cholinergic innervation in guinea pig heart by immunohistochemistry for high-affinity choline transporters. Cardiovasc Res 2004;62:112-21.

24. Scherer-Singler U, Vincent SR, Kimura H, Mc-Geer EG. Demonstration of a unique population of neurons with NADPHdiaphorase histochemistry. J Neurosci Methods 1983;9:229-34.

25. Kluchova D, Klimcik R, Kloc P. Neuronal nitric oxide synthase in the rabbit spinal cord visualised by histochemical NADPH-diaphorase and immunohistochemical NOS methods. Gen Physiol Biophys 2002;21:163-74.

26. El-Badawi A, Schenk E. Histochemical methods for separate, consecutive and simultaneous demonstrations of acetylcholinesterase and norepinephrine in cryostat sections. J Histochem Cytochem 1967;15:580-8.

27. Kukanova B, Mravec, B. Complex intracardiac nervous system. Bratisl Lek Listy 2006;107:45-51.

28. Oki T, Fukuda N, Kawano $\mathrm{T}$ et al. Histopathologic studies of innervation of normal and prolapsed human mitral valves. J Heart Valve Dis 1995;4:496-502.

29. Jew JY, Williams TH. Innervation of the mitral valve is strikingly depleted with age. Anat Rec 1999;255:252-60.

30. Kumar SD, Tay SSW. Changes in peptidergic nerves in the atrioventricular valves of streptozotocin-induced diabetic rats: A confocal microscopic study. Anat Rec 2000;258:277-85.

31. Crick SJ, Anderson RH, Ho SY, Sheppard MN. Localization and quantitation of autonomic innervation in the porcine heart II: endocardium, myocardium and epicardium. J Anat 1999;195:359-73.

32. Marron K, Yacoub MH, Polak JM et al. Innervation of human atrioventricular and arterial valves. Circulation 1996;94:368-75.

33. Ahmed A, Johansson 0, Folan-Curran J. Distribution of PGP 9.5, TH, NPY, SP and CGRP immunoreactive nerves in the rat and guinea pig atrioventricular valves and chordae tendineae. J Anat 1997;191:54760 .

34. Singh S, Johnson PI, Javed A et al. Monoamine-and histamine synthesizing enzymes and neurotransmitters within neurons of adult human cardiac ganglia. Circulation 1999;99:411-9.

35. Shoba T, Tay SS. Nitrergic and peptidergic innervation in the developing rat heart. Anat Embryol 2000;201:491-500.

36. Maifrino LBM, Liberti EA, Castelucci P, de Souza RR. NAPH-diaphorase positive cardiac neurons in the atria of mice. A morphoquantitative study. BMC Neurosci 2006;2:7-10.

37. Pauziene N, Pauza DH, Stropus R. Morphology of human intracardiac nerves: an electron microscope study. J Anat 2000;19:437-60.

38. Andries LJ, Brutsaert DL. Endocardial endothelium in the rat: cell shape and organization of the cytoskeleton. Cell Tissue Res 1998;273:107-17.

39. Noria S, Cowan DB, Gotlieb AI, Langille
BL. Transient and steady-state effects of shear stress on endothelial cell adherens junctions. Circ Res 1999;85:504-14.

40. Sawada K, Kondo T, Chang J et al. Distribution and neuropeptide content of nitric oxide synthase-containing nerve fibers in arteries and conduction system of the rat heart. Acta Anat 1997;160:239-47.

41. Antzelevitch Ch, Burashnikov A, Di Diego JM. Mechanisms of cardiac arrhythmia. In: Gussak I, Antzelevitch Ch, Wilde AAM, Friedman PA, Ackerman MJ, Shen WK. Electrical Diseases of the Heart. Genetics, Mechanisms, Treatment, Prevention. Part I. Springer, London, 2008, pp. 65-132.

42. Williams TH, Folan JC, Jew JY, Wang YF. Variations in atrioventricular valve innervation in four species of mammals. Am J Anat 1990;187:193-200.

43. Marron K, Wharton J, Sheppard MN et al. Distribution, morphology and neurochemistry of endocardial and epicardial nerve terminal arborizations in the human heart. Circulation 1995;92:2343-51.

44. Herring N, Danson EJF, Paterson DJ. Cholinergic control of heart rate by nitric oxide is site specific. News Physiol Sci 2002;17:202-6.

45. Sartori C, Lepori M, Scherrer U. Interaction between nitric oxide and the cholinergic and sympathetic nervous system in cardiovascular control in humans. Pharmacol Ther 2005;106:209-20.

46. Yoshida K, Toda N. NADPH diaphorasepositive neurons in the intracardiac plexus of human, monkey and canine right atria. Brain Res 1996;724:256-9. 